\title{
Probing the inner wind of AGB stars: Interferometric observations of SiO millimetre line emission from the oxygen-rich stars $R$ Dor and $L^{2}$ Pup
}

\author{
F. L. Schöier ${ }^{1}$, H. Olofsson ${ }^{1}$, T. Wong ${ }^{2,3}$, M. Lindqvist ${ }^{4}$, and F. Kerschbaum ${ }^{5}$ \\ 1 Stockholm Observatory, AlbaNova, 10691 Stockholm, Sweden \\ e-mail: fredrik@astro.su.se \\ 2 CSIRO Australia Telescope National Facility, PO Box 76, Epping NSW 1710, Australia \\ 3 School of Physics, University of New South Wales, Sydney NSW 2052, Australia \\ ${ }^{4}$ Onsala Space Observatory, 43992 Onsala, Sweden \\ 5 Institut für Astronomie, Türkenschanzstraße 17, 1180 Wien, Austria \\ Received 19 March 2004 / Accepted 8 April 2004
}

\begin{abstract}
High angular resolution Australia Telescope Compact Array (ATCA) observations of SiO "thermal" millimetre line emission towards the two oxygen-rich, low mass loss rate AGB stars R Dor and $\mathrm{L}^{2}$ Pup are presented. In both cases the emission is resolved with an overall spherical symmetry. Detailed radiative transfer modelling of the SiO line emission has been performed, and the comparison between observations and models are conducted in the visibility plane, maximizing the sensitivity. The excitation analysis suggests that the abundance of $\mathrm{SiO}$ is as high as $4 \times 10^{-5}$ in the inner part of the wind, close to the predicted values from stellar atmosphere models. Beyond a radius of $\approx 1 \times 10^{15} \mathrm{~cm}$ the $\mathrm{SiO}$ abundance is significantly lower, about $3 \times 10^{-6}$, until it decreases strongly at a radius of about $3 \times 10^{15} \mathrm{~cm}$. This is consistent with a scenario where $\mathrm{SiO}$ first freezes out onto dust grains, and then eventually becomes photodissociated by the interstellar UV-radiation field. In these low expansion velocity sources the turbulent broadening of the lines plays an important role in the line formation. Microturbulent velocity widths in the range $1.1-1.5 \mathrm{~km} \mathrm{~s}^{-1}$ result in a very good reproduction of the observed line shapes even if the gas expansion velocity is kept constant. This, combined with the fact that the $\mathrm{SiO}$ and $\mathrm{CO}$ lines are well fitted using the same gas expansion velocity (to within 5-10\%), suggest that the envelope acceleration occurs close to the stellar photosphere, within $\$ 20-30$ stellar radii.
\end{abstract}

Key words. stars: AGB and post-AGB - stars: carbon - stars: late-type - stars: mass-loss

\section{Introduction}

The intense winds that low to intermediate mass stars develop in their final evolutionary stage, as they ascend the asymptotic giant branch (AGB), return enriched stellar material to the interstellar medium. In addition to significantly contributing to the chemical evolution of galaxies, the mass loss will dictate the time scale for the future evolution of the star towards the planetary nebula phase. Considering its importance little is known with certainty about the mechanism(s) behind the mass loss. Partly this is due to a lack of observational constraints, in particular close to the stellar photosphere where the wind is accelerated, and partly due to the complexity of the physical/chemical processes involved. Among other things, the formation of dust grains is thought to play an important role in these radiatively driven winds. Most of the information on this region comes from observations of infrared ro-vibrational molecular lines in absorption, mainly towards the carbon star IRC+10216 (e.g., Keady \& Ridgway 1993; Winters et al. 2000a), infrared continuum emission from the circumstellar dust (e.g., Danchi et al. 1994), and SiO maser radio line emission from excited vibrational states (e.g., Cotton et al. 2004).

Information can also be gained from radio observations of suitable circumstellar molecular species towards stars having low to intermediate mass loss rates. A major survey of $\mathrm{CO}$ radio line emission from circumstellar envelopes (CSEs) around oxygen-rich AGB stars of different variability types were done by Kerschbaum \& Olofsson (1999) and Olofsson et al. (2002). These data were modelled in detail to derive stellar mass loss rates and terminal gas expansion velocities (Olofsson et al. 2002). Subsequently, a survey of "thermal" SiO radio line emission, meaning emission from the ground vibrational state rotational lines which are normally not (strongly) masering, was done and the data were interpreted using a detailed numerical radiative transfer modelling presented in González Delgado et al. (2003).

An immediate conclusion from these large surveys is that the $\mathrm{SiO}$ and $\mathrm{CO}$ radio line profiles are different from each other. The SiO line profiles are narrower in the sense that the 
main fraction of the emission comes from a velocity range smaller by about $10-20 \%$ than twice the expansion velocity determined from the $\mathrm{CO}$ data. On the other hand, the $\mathrm{SiO}$ line profiles have weak wings, such that the total velocity width of its emission is very similar to that of the $\mathrm{CO}$ emission. Furthermore, it appears that the SiO line profiles change character with the mass loss rate, at low mass loss rates they are narrow with weak extended wings, while at high mass loss rates they become distinctly triangular. These "peculiar" SiO line profiles have been interpreted as being due to the influence of gas acceleration in the region which produces most of the $\mathrm{SiO}$ line emission (Bujarrabal et al. 1986). However, as illustrated in González Delgado et al. (2003), the SiO lines are usually strongly self-absorbed also for low mass loss rate objects and this produces narrower lines. There is some controversy in the literature over the scale length of the acceleration region. In the only previously published works where thermal $\mathrm{SiO}$ emission have been observed towards AGB stars using interferometry, Sahai \& Bieging (1993) find no need for the slowly varying velocity fields introduced by Lucas et al. (1992).

Other conclusions come from the detailed radiative transfer modelling of the $\mathrm{SiO}$ line data. This is in many respects a more difficult enterprise than the $\mathrm{CO}$ line modelling. The $\mathrm{SiO}$ line emission predominantly comes from a region closer to the star than does the $\mathrm{CO}$ line emission, and this is a region where we have fewer observational constraints. The $\mathrm{SiO}$ excitation is also normally far from thermal equilibrium with the gas kinetic temperature, and radiative excitation plays a major role. Finally, there exists no detailed chemical model for calculating the radial $\mathrm{SiO}$ abundance distribution. González Delgado et al. (2003) adopted the assumption that the gas-phase SiO abundance stays high only very close to the star, since further out the $\mathrm{SiO}$ molecules are adsorbed onto the grains. Beyond this the abundance stays low until the molecules are eventually dissociated by the interstellar UV radiation. This photodissociation radius, which is crucial to the modelling, was estimated using both $\mathrm{SiO}$ multi-line modelling and existing interferometer data (Lucas et al. 1992; Sahai \& Bieging 1993), but only for a few sources. The result of the radiative transfer modelling is a circumstellar $\mathrm{SiO}$ abundance that is roughly the same as that obtained from stellar atmosphere equilibrium chemistry for low mass loss rate objects, and which declines with mass loss rate reaching an abundance about two orders of magnitude lower for high mass loss rate objects.

Thus, there are strong indications that "thermal" $\mathrm{SiO}$ radio line emission is a useful probe of the formation and evolution of dust grains in a CSE, as well as of its dynamics. Hence, circumstellar $\mathrm{SiO}$ line emission potentially carries information on the properties of the region where the mass loss of AGB stars is initiated.

Presented here are results from high spatial resolution imaging of the two M-type semiregular AGB stars R Dor and $\mathrm{L}^{2}$ Pup. Both sources studied have low mass loss rates $\left(\dot{M} \lesssim 1 \times 10^{-7} M_{\odot} \mathrm{yr}^{-1}\right)$ and low terminal velocities of their winds $\left(\$ 6 \mathrm{~km} \mathrm{~s}^{-1}\right)$. It has been suggested from hydrodynamical calculations (Bowen 1988; Winters et al. 2000b, 2002, 2003; Struck et al. 2004) that the main driving mechanism behind these tenuous winds is stellar pulsation and that dust plays only a secondary role.

The observations, performed with the Australia Telescope Compact Array ${ }^{1}$ (ATCA), are presented in Sect. 2. ATCA is an array of six $22 \mathrm{~m}$ dishes operating from $1.4-26 \mathrm{GHz}$ and with an upgrade to $85-105 \mathrm{GHz}$ operation in progress. Its location makes it a unique instrument to study molecular-line sources in the southern hemisphere at high angular resolution, and we have recently used it to conduct a study of circumstellar $\mathrm{HCN}$ emission from the carbon star R Scl (Wong et al. 2004). The analysis in Sect. 3 and comparison with envelope models in Sect. 4 are carried out in the $u v$-plane in order to maximize the sensitivity and resolution of the data. The modelling is followed by a discussion in Sect. 5 and conclusions are presented in Sect. 6.

\section{Observations and data reduction}

At the time of the observations, the ATCA had three antennas of $22 \mathrm{~m}$ diameter equipped with dual polarisation $3-\mathrm{mm}$ receivers covering the bands $84.9-87.3$ and $88.5-91.3 \mathrm{GHz}$. We observed R Dor and L 2 Pup between August and October 2003 in three different array configurations: EW367 (baselines of 45, 90, and $135 \mathrm{~m}), 750 \mathrm{~B}(60,165$, and $225 \mathrm{~m})$, and $\mathrm{H} 214$ (75, 135 , and $210 \mathrm{~m}$ ). EW367 and 750B provided east-west baselines whereas $\mathrm{H} 214$ provided north-south baselines. The observations in each configuration occurred on single nights. The combined visibility plane coverage is shown in Fig. 1.

All observations were conducted in clear to partly cloudy weather, with above-atmosphere single-sideband system temperatures of $T_{\text {sys }}^{*} \approx 350-500 \mathrm{~K}$ near the zenith. The correlator was configured to receive both linear polarisations in two frequency windows: a narrowband (spectral line) window centred on the "thermal" $\mathrm{SiO} v=0, J=2 \rightarrow 1$ line at $86.847 \mathrm{GHz}$ with 128 channels across $32 \mathrm{MHz}$, and a wideband window centred on the "maser" $\mathrm{SiO} v=1, J=2 \rightarrow 1$ line at $86.243 \mathrm{GHz}$ with 32 channels across $128 \mathrm{MHz}$. The pointing and phase centre was at $\alpha_{2000}=04^{\mathrm{h}} 36^{\mathrm{m}} 45^{\mathrm{s}} .67, \delta_{2000}=-62^{\circ} 04^{\prime} 37^{\prime \prime} \cdot 9$ for R Dor and at $\alpha_{2000}=07^{\mathrm{h}} 13^{\mathrm{m}} 32^{\mathrm{s}} .31, \delta_{2000}=-44^{\circ} 38^{\prime} 24^{\prime \prime} .1$ for $\mathrm{L}^{2}$ Pup. The field of view is determined by the ATCA primary beam which has a FWHM of about $36^{\prime \prime}$ at $86 \mathrm{GHz}$.

As an initial step in the reduction of the $750 \mathrm{~B}$ and H214 data, an elevation-dependent gain curve was applied based on observations of an $\mathrm{SiO}$ maser taken on 2003 Sep. 4. No such gain curve was available for the earlier EW367 observation. Gain calibration as a function of time was then performed by assuming the $\mathrm{SiO}$ maser emission comes from a point source located at the phase centre. Since the maser emission is known to emanate from a region only a few $\mathrm{AU}$ in radius (e.g., Cotton et al. 2004), this is a reasonable assumption for our resolution of a few arcseconds ( $1 \mathrm{AU}=20$ milliarcsec at the distance of our nearest source, $\mathrm{R}$ Dor). To derive the amplitude gains we also assumed that the integrated $\mathrm{SiO}$ maser flux was constant throughout each observation. We found that the variation in the gain was generally dominated by an uncorrected

1 The Australia Telescope is funded by the Commonwealth of Australia for operation as a National Facility managed by CSIRO. 

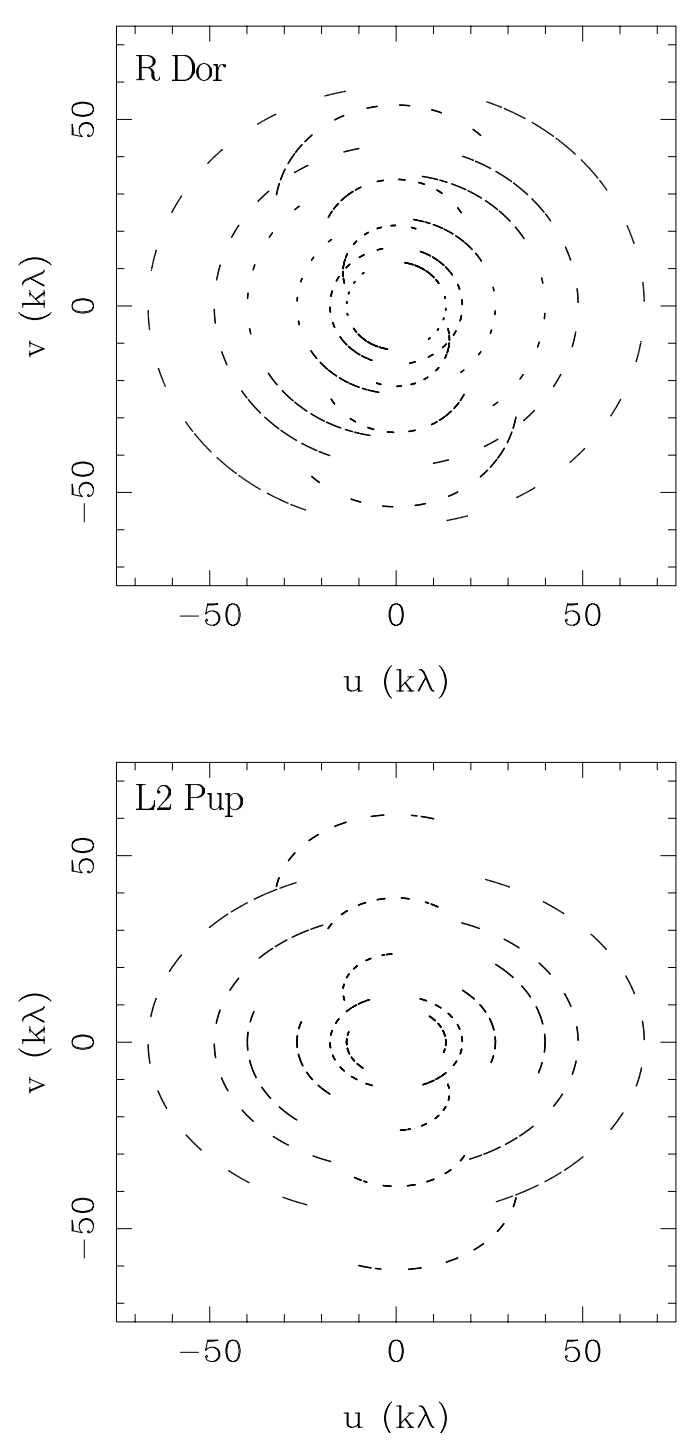

Fig. 1. Coverage of the visibility plane for $R$ Dor and $\mathrm{L}^{2}$ Pup obtained after combining the three ATCA configurations used (EW367, 750B, and $\mathrm{H} 214$ ).

gain-elevation dependence, limiting any intrinsic flux variation to $\$ 10 \%$.

The amplitude and phase gains were transferred from the maser to the spectral line window after applying scaling factors determined from a half-hour integration on the bandpass calibrator (3C 279 or PKS B1921-293), which was also used to determine the channel-dependent gains. In the case of $\mathrm{L}^{2} \mathrm{Pup}$, the transfer of the phase gains could not be performed successfully because of an inconsistency in the frequency setup between the source and bandpass calibrator; consequently we have applied phase self-calibration to the thermal $\mathrm{SiO}$ emission from this source assuming that it is circularly symmetric. For an interferometer the astrometry is determined by the quality of the phase calibration. For R Dor the phase errors were $\sim 5^{\circ}$ and one would expect the position error to be about $0.1^{\prime \prime}$ for a $100 \mathrm{~m}$ baseline.

Uranus was used to set the flux scale, assuming a uniform disk with brightness temperature of $134 \mathrm{~K}$. However, for the EW367 array, where the Uranus observation occurred in poor weather, the flux scale was tied to that of the 750B observation by assuming the $\mathrm{SiO}$ maser fluxes from both sources were unchanged over the 17-day gap between the observations. Assuming that any actual flux variations for the two sources were uncorrelated, the derived gains suggest an uncertainty in the flux scale for this configuration of $\approx 30 \%$. We also adjusted the antenna pointing once an hour on the $\mathrm{SiO}$ masers; typical pointing shifts were $\approx 5^{\prime \prime}$.

All data processing was conducted using the MIRIAD package (Sault et al. 1995). The calibrated visibilities were Fourier transformed using uniform weighting and a 0.5 pixel size with a channel spacing of $1 \mathrm{~km} \mathrm{~s}^{-1}$ (roughly the effective velocity resolution given the original channel spacing of $0.25 \mathrm{MHz} \approx 0.8 \mathrm{~km} \mathrm{~s}^{-1}$ ). The maps were then CLEANed (Högbom 1974) down to a $2 \sigma$ level over the inner $20^{\prime \prime} \times 20^{\prime \prime}$.

The actual analysis and comparison with the model will be carried out in the $u v$-plane to maximize the sensitivity and resolution of the data. Thus we expect to obtain usable information on scales as low as $1^{\prime \prime}$, corresponding to the longest baselines.

\section{Observational results}

\section{1. $R$ Dor}

The $\mathrm{SiO} v=0, J=2 \rightarrow 1$ velocity channel maps of R Dor, binned into $2 \mathrm{~km} \mathrm{~s}^{-1}$ channels, are shown in Fig. 2. The synthesised beam is $2^{\prime \prime} 6 \times 22^{\prime \prime} 0$ with a position angle of $-56^{\circ}$ and the rms noise in the $1 \mathrm{~km} \mathrm{~s}^{-1}$ velocity channel maps is $\sigma=30$ mJy beam $^{-1}$, as estimated from regions free of source emission. The signal-to-noise ratio is high with the peak emission reaching a level of about $300 \sigma$.

The velocity channel maps suggest that the $\mathrm{SiO} v=0, J=$ $2 \rightarrow 1$ emission towards $\mathrm{R}$ Dor is moderately resolved, a result which is confirmed by the visibility analysis (see below). The brightness distribution appears to have an overall circular symmetry. From fitting two-dimensional Gaussian brightness distributions to the individual channel maps, it is concluded that no systematic variations in position with velocity are present. The individual offsets are smaller than 0.1 , within the pointing uncertainty (Sect. 2), confirming the adopted source position. Across the peak of the line profile, from $6-8 \mathrm{~km} \mathrm{~s}^{-1}$ (LSR), the average values of the major and minor axes are 3 .'3 and 3.'7 (FWHM), respectively. The beam has not been deconvolved from these values.

As interferometers lacks sensitivity to large scale emission it is of interest to find out whether part of the emission is resolved out in the ATCA data. In Fig. 3 the ATCA SiO data has been restored with a 57" circular beam (solid line; this represents the beam of the SwedishESO Submillimetre Telescope, SEST). A comparison with the equivalent SEST spectrum from González Delgado et al. (2003) (histogram) shows a striking resemblance both with regard to the peak flux ( $\approx 20 \mathrm{Jy})$ and the overall line shape. This strongly suggests that all the flux is recovered by the ATCA interferometer, at least within the 57" SEST beam, and that virtually all of the $\mathrm{SiO}$ emission comes from the central 


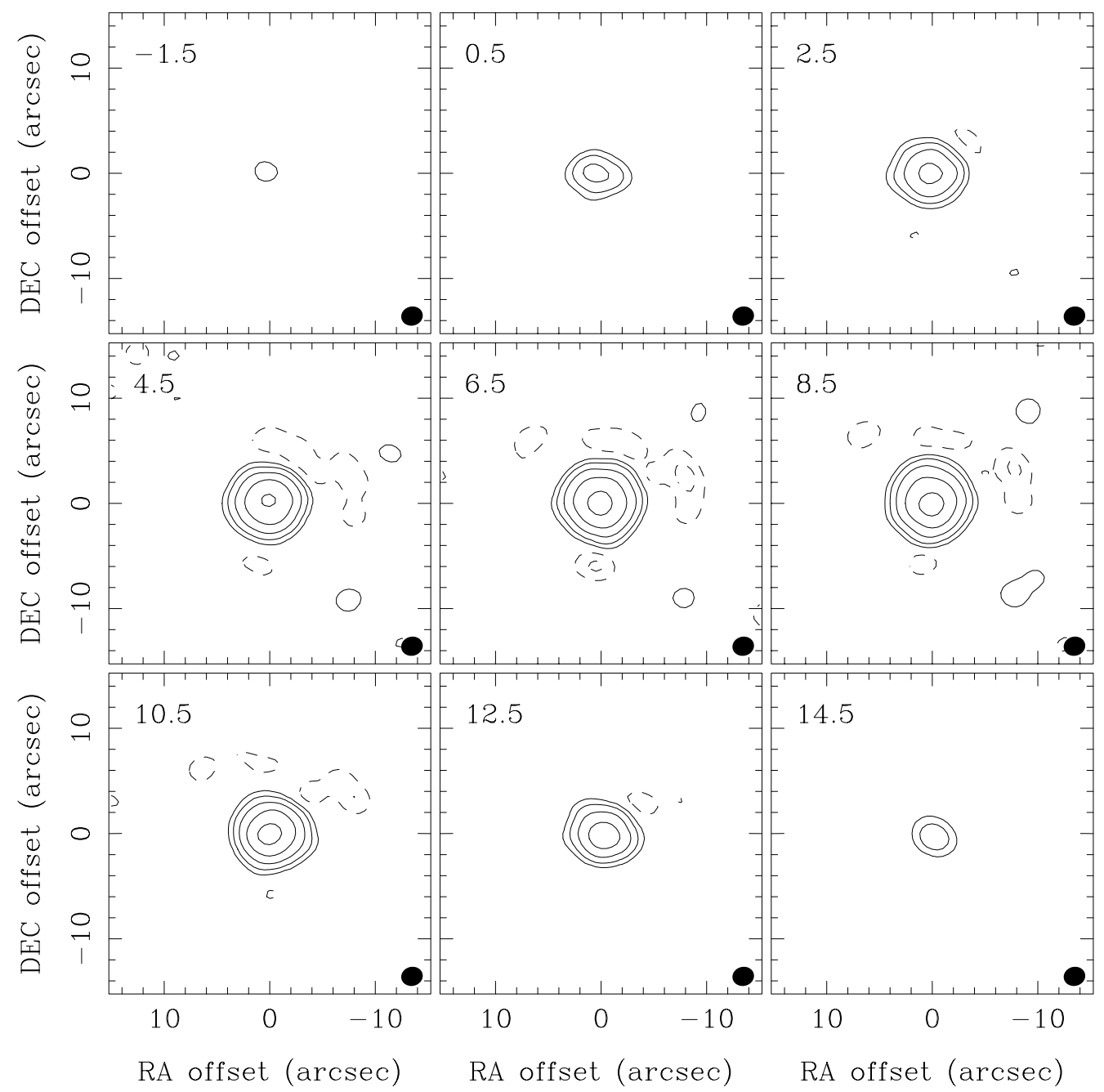

Fig. 2. Velocity channel maps of $\mathrm{SiO} v=0, J=2 \rightarrow 1$ emission from $\mathrm{R}$ Dor. The contour levels are $0.03 n \mathrm{Jy}^{\text {beam }}{ }^{-1}$, where $n=-9,-3,3,9,27,81,243$ (negative values have dashed contours), and the beam size is $2^{\prime \prime} .6 \times 22^{\prime \prime} 0$ with a position angle of $-56^{\circ}$ as indicated in the lower right hand corner of each panel. The velocity channels (given in the LSR frame and indicated in the upper left corner) have been binned to $2 \mathrm{~km} \mathrm{~s}^{-1}$. The systemic velocity is $7 \mathrm{~km} \mathrm{~s}^{-1}$ as determined from $\mathrm{CO}$ observations. Offsets in position are relative to $\alpha_{2000}=04^{\mathrm{h}} 36^{\mathrm{m}} 45^{\mathrm{s}} \cdot 67, \delta_{2000}=-62^{\circ} 04^{\prime} 37^{\prime \prime} \cdot 9$.

component. The SEST spectrum has been converted from main beam brightness temperature scale to Jy using

$S=\eta_{\mathrm{mb}} \Gamma^{-1} T_{\mathrm{mb}}$

where the main-beam efficiency $\eta_{\mathrm{mb}}=0.75$ and the sensitivity $\Gamma^{-1}=25 \mathrm{Jy} \mathrm{K}^{-1}$. The telescope parameters are taken from the SEST homepage ${ }^{2}$.

Assuming that the emission has an overall spherical symmetry, circularly symmetric Gaussians have been fitted fitted to the visibilities. Given the higher sensitivity in the $u v$-plane there appears to be a systematic trend with velocity as shown in Fig. 4. The largest offsets are measured at the extreme velocities, i.e., the blue- and redshifted emissions are displaced from each other. Lindqvist et al. (2000), when analysing several molecular line emissions from the high mass loss rate carbon star CIT 6, also noted that there was a common trend in position with velocity. A reasonable explanation for this behaviour

\footnotetext{
${ }^{2}$ www.ls.eso.org/lasilla/Telescopes/SEST/SEST.html
}

is that the CSE is asymmetric to some degree, perhaps a bipolar outflow, but the present angular resolution is not enough to make this apparent in the channel maps, Fig. 2.

The resulting $F W H M$ of Gaussians fitted to the azimuthally averaged visibilities are plotted in Fig. 5. The overall variation in size with line-of-sight velocity is as expected for a well resolved expanding envelope, where gas moving orthogonal to the line of sight subtends a larger solid angle than radially moving gas near the extreme velocities. The largest angular extent of the emission is $33^{\prime \prime} 0 \pm 0.2$ in the velocity range $6-8 \mathrm{~km} \mathrm{~s}^{-1}$ (LSR). This corresponds to a radial size of $1.0 \times 10^{15} \mathrm{~cm}$ at the distance of $45 \mathrm{pc}$. We note that a single Gaussian provides a reasonable fit to the visibilities on all baselines except those close to the systemic velocity at $7 \mathrm{~km} \mathrm{~s}^{-1}$ (LSR). Introducing an offset in the flux scale, representing an unresolved additional component, significantly improves the fit at those velocities. In Sect. 4.3 it is shown that the visiblilities obtained for this source are best fitted using two 


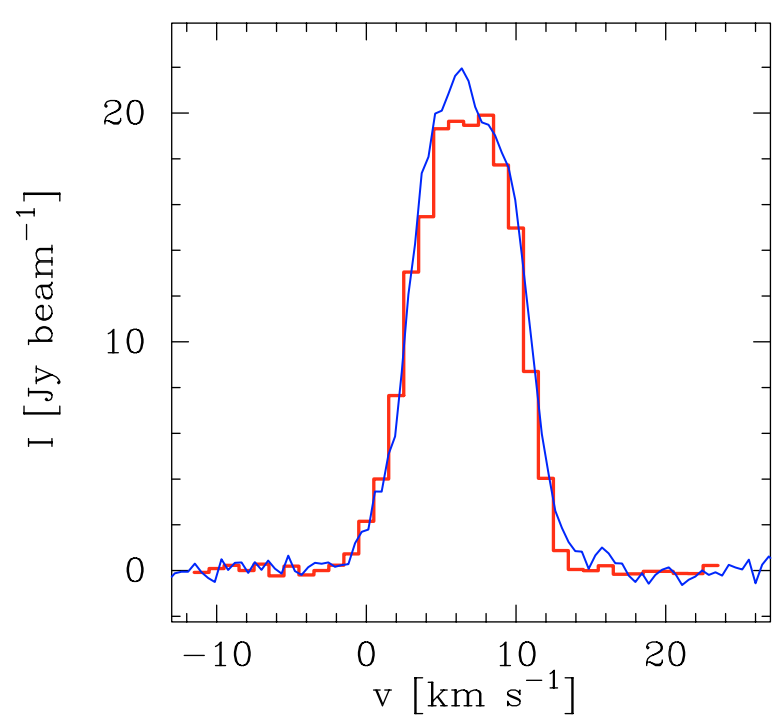

Fig. 3. $\mathrm{SiO} v=0, J=2 \rightarrow 1$ spectra for R Dor. The solid line is the SEST single dish observation (González Delgado et al. 2003), whereas the histogram shows the spectrum at the phase centre derived from the ATCA data by restoring with the SEST beam of 57".

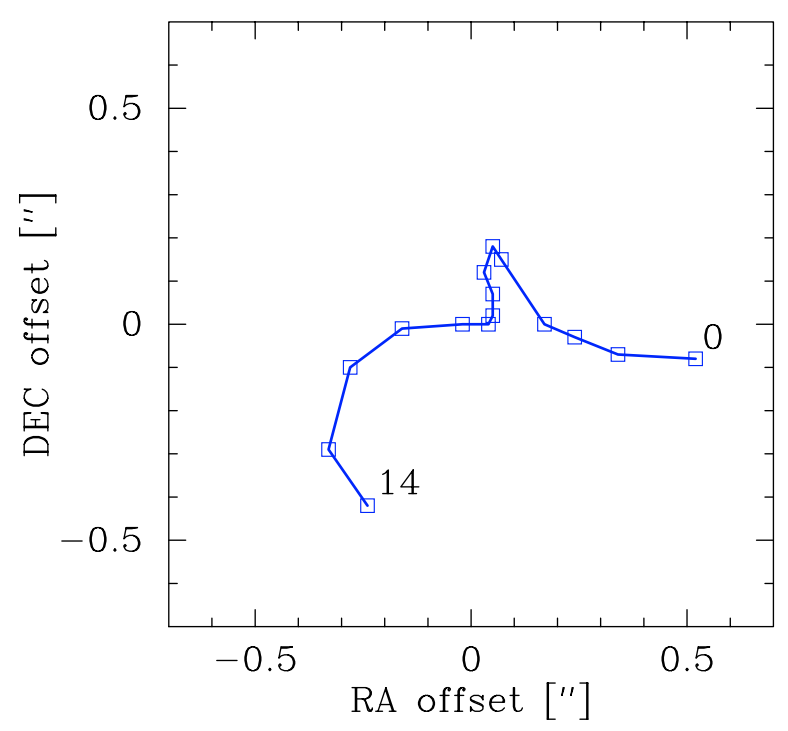

Fig. 4. Offset from the phase centre of the velocity channels as determined from fitting circular Gaussians to the R Dor data in the $u v$ plane. Indicated are the velocities of the two extreme channels ( 0 and $14 \mathrm{~km} \mathrm{~s}^{-1}$ ) for which reliable fits could be obtained.

components: one compact with high a $\mathrm{SiO}$ abundance and one more extended with a significantly lower abundance.

\section{2. $L^{2}$ Pup}

The $\mathrm{SiO} v=0, J=2 \rightarrow 1$ velocity channel maps of $\mathrm{L}^{2} \mathrm{Pup}$, binned into $2 \mathrm{~km} \mathrm{~s}^{-1}$ channels, are shown in Fig. 6 . The synthesised beam is $2^{\prime \prime} .5 \times 1^{\prime \prime}$. 9 with a position angle of $14^{\circ}$ and the rms noise in the $1 \mathrm{~km} \mathrm{~s}^{-1}$ velocity channel maps is $\sigma=$ $40 \mathrm{mJy} \mathrm{beam}^{-1}$, as estimated from regions free of source emission. The signal-to-noise ratio is high with the peak emission reaching a level of about $60 \sigma$.

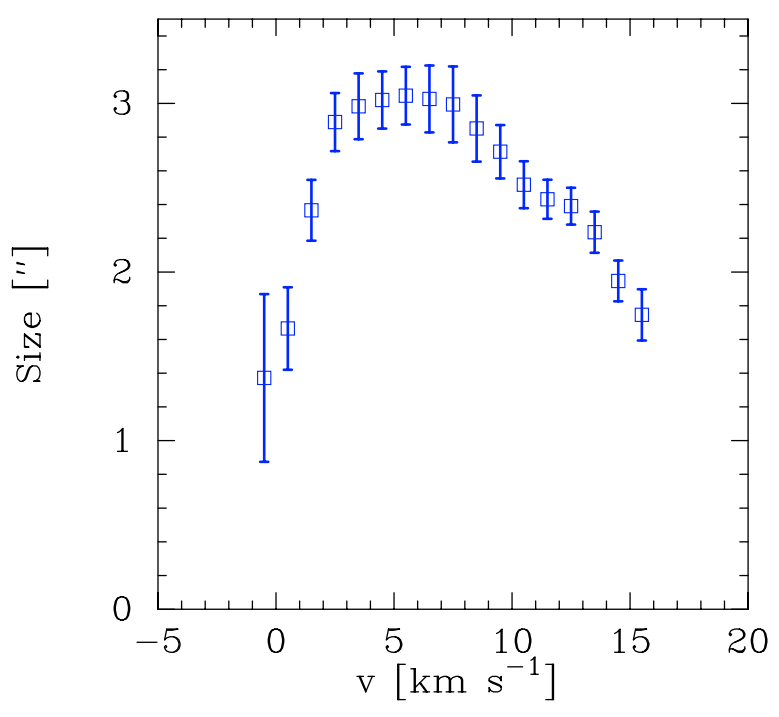

Fig. 5. Size $(F W H M)$ of the observed $\mathrm{SiO}$ emission toward $\mathrm{R}$ Dor as function of velocity channel (given in LSR frame), averaged in $1 \mathrm{~km} \mathrm{~s}^{-1}$ bins, as estimated from Gaussian fits to the azimuthally averaged visibility data.

As expected from the single-dish modelling performed by González Delgado et al. (2003) L 2 Pup has a smaller envelope than R Dor. Since we had to self-calibrate the data in phase for this object, we have assumed that the source distribution is symmetric and centred on the stellar position. Across the peak of the line profile, from 33 to $35 \mathrm{~km} \mathrm{~s}^{-1}$ (LSR), the average values of the major and minor axes are 2.9 and 2.2 (FWHM), respectively (note that the beam has not been deconvolved from these values).

In Fig. 7 the ATCA $\mathrm{SiO}$ data for $\mathrm{L}^{2}$ Pup has been restored with a 57" circular beam (solid line), representing the SEST beam. A comparison with the equivalent SEST spectrum of González Delgado et al. (2003) (histogram; converted from main beam brightness temperature scale to Jy using Eq. (1)) shows a striking resemblance both with regard to the peak flux $(\approx 3 \mathrm{Jy})$ and the overall line shape. As for R Dor this strongly suggests that all the flux is recovered by the ATCA interferometer, and that all of the $\mathrm{SiO}$ emission comes from the central compact component.

The result of fitting Gaussians to the azimuthally averaged visibilities is presented in Fig. 8. The variation in size is also here consistent with a resolved expanding envelope. The largest angular extent of the emission is $11^{\prime \prime} 6 \pm 0$ ". 1 in the velocity range $33-35 \mathrm{~km} \mathrm{~s}^{-1}$ (LSR). This corresponds to a radial size of $1.0 \times$ $10^{15} \mathrm{~cm}$ at the distance of $85 \mathrm{pc}$. Contrary to R Dor, a Gaussian gives a reasonably good description of the visibilities on all baselines and for all velocity channels.

\section{Radiative transfer modelling}

A detailed non-LTE radiative transfer code, based on the Monte Carlo method (Bernes 1979), is used to perform the excitation analysis and model the observed circumstellar line emission. The code is described in detail in Schöier \& Olofsson (2001) and has been benchmarked, to high accuracy, against a wide 


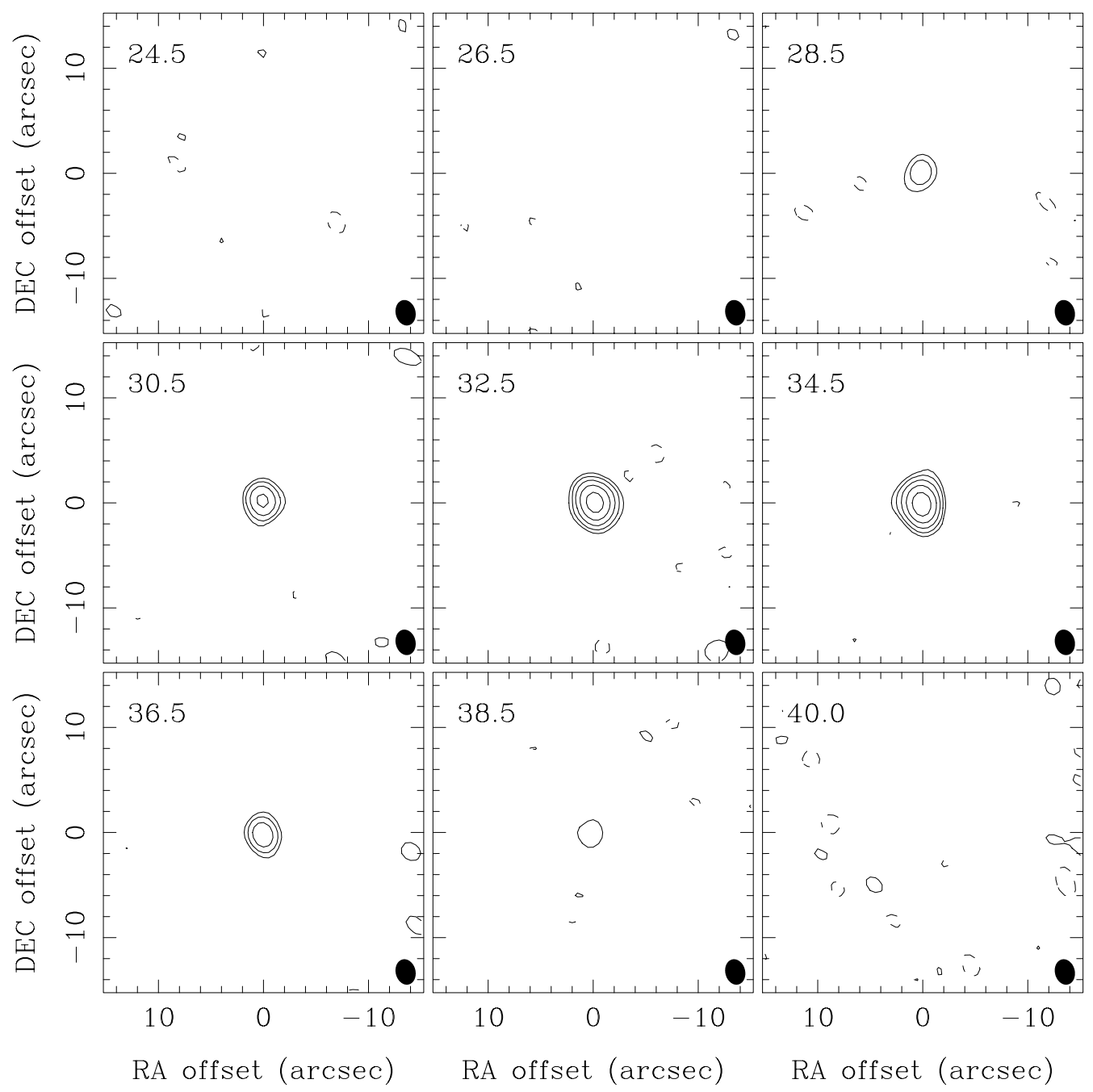

Fig. 6. Velocity channel maps of $\mathrm{SiO} v=0, J=2 \rightarrow 1$ emission from $\mathrm{L}^{2}$ Pup. The contour levels are $0.04 n \mathrm{Jy}$ beam ${ }^{-1}$, where $n=-2,2,4,8,16,32$ (negative values have dashed contours), and the beam size is $2{ }^{\prime \prime} .5 \times 11^{\prime \prime} 9$ with a position angle of $14^{\circ}$ as indicated in the lower right hand corner of each panel. The velocity channels (given in the LSR frame and indicated in the upper left corner) have been binned to $2 \mathrm{~km} \mathrm{~s}^{-1}$. The systemic velocity is $33 \mathrm{~km} \mathrm{~s}^{-1}$ as determined from CO observations. Offsets in position are relative to $\alpha_{2000}=07^{\mathrm{h}} 13^{\mathrm{m}} 32^{\mathrm{s}} \cdot 31$, $\delta_{2000}=-44^{\circ} 38^{\prime} 24^{\prime \prime} .1$.

variety of molecular line radiative transfer codes in van Zadelhoff et al. (2002).

\subsection{The physical structure of the CSE}

The CSEs are assumed to be spherically symmetric and to expand at constant velocity, and they are produced by constant mass loss rates. Both $\mathrm{R}$ Dor and $\mathrm{L}^{2}$ Pup were included in the large survey of semiregular M-type AGB-variables by Kerschbaum \& Olofsson (1999). Their overall wind properties such as mass loss rate, terminal expansion velocity and temperature structure as constrained by single-dish $\mathrm{CO}$ millimetre line emission were presented in Olofsson et al. (2002), where also a more detailed description of the circumstellar model is given.

Olofsson et al. (2002) noted that the CO lines for $\mathrm{L}^{2}$ Pup are better reproduced when adopting a higher value of $1.0 \mathrm{~km} \mathrm{~s}^{-1}$ for the turbulent velocity than the nominal value of $0.5 \mathrm{~km} \mathrm{~s}^{-1}$. Further support for a larger turbulent velocity are the $\mathrm{SiO}$ line wings that are commonly observed in the low expansion velocity sources such as R Dor and $\mathrm{L}^{2}$ Pup (González Delgado et al. 2003). Therefore, in the present analysis the turbulent velocity width is used as a free parameter.

Olofsson et al. (2002) calculated the kinetic temperature distribution including, e.g., CO line cooling. Here we add cooling by $\mathrm{H}_{2} \mathrm{O}$ since this may be of importance in the region where the $\mathrm{SiO}$ line emission is produced (González Delgado et al. 2003). The importance of $\mathrm{H}_{2} \mathrm{O}$ as a coolant in the inner wind was also noted by Zubko \& Elitzur (2000) in their study of W Hya. In order to be consistent we have therefore produced new circumstellar models for also the CO line emission. The inclusion of $\mathrm{H}_{2} \mathrm{O}$ line cooling lowers the temperature significantly (from 15-40\%) in the inner part $\left(r \lesssim 1 \times 10^{15} \mathrm{~cm}\right.$ ) of the envelope, but has only a minor effect on the mass loss rate derived from $\mathrm{CO}$ observations. More details on how the $\mathrm{H}_{2} \mathrm{O}$ line cooling is treated, and its effect on $\mathrm{CO}$ and $\mathrm{SiO}$ line intensities, are given in Olofsson et al. (2002) and González Delgado et al. (2003). As an example, we note that excluding $\mathrm{H}_{2} \mathrm{O}$ line 


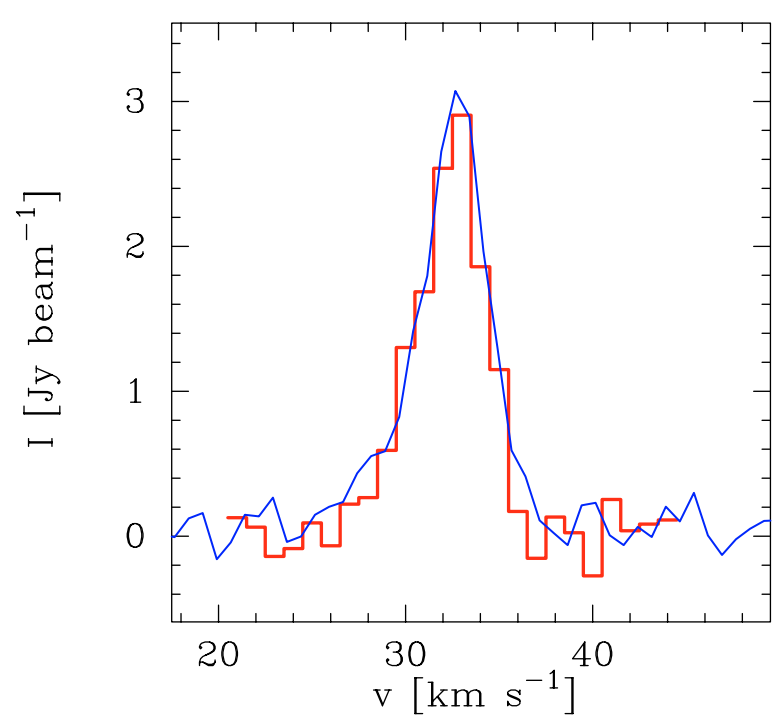

Fig. 7. $\mathrm{SiO} v=0, J=2 \rightarrow 1$ spectra for $\mathrm{L}^{2}$ Pup. The solid line is the SEST single dish observation (González Delgado et al. 2003), whereas the histogram shows the spectrum at the phase centre derived from the ATCA data by restoring with the SEST beam of 57".

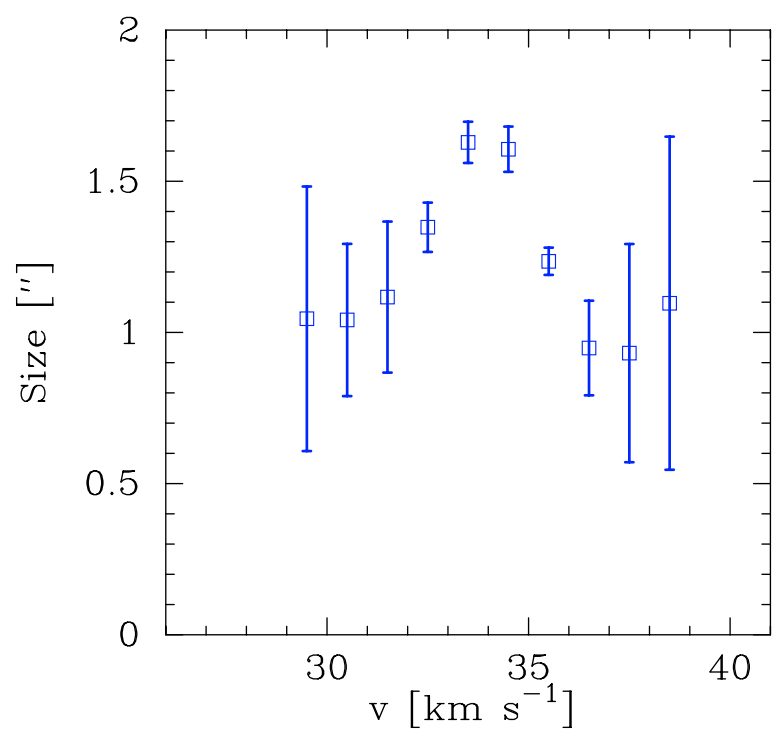

Fig. 8. Size $(F W H M)$ of the observed $\mathrm{SiO}$ emission toward $\mathrm{L}^{2}$ Pup as function of velocity channel (given in LSR frame), averaged in $1 \mathrm{~km} \mathrm{~s}^{-1}$ bins, as estimated from Gaussian fits to the azimuthally averaged visibility data.

cooling increases the predicted $\mathrm{SiO}$ line intensities in our best fit model for R Dor (presented in Sect. 4.3) by $10-15 \%$ for the $J=5 \rightarrow 4$ and $J=6 \rightarrow 5$ SEST observations as well as the $J=2 \rightarrow 1$ interferometric ATCA observations. These are the data most sensitive to the physical and chemical conditions prevailing in the inner wind. For comparison, the predicted SEST $J=2 \rightarrow 1$ and $J=3 \rightarrow 2$ line intensities only increase by a few $\%$.

We find that the molecular line emission is better reproduced (in particular that of $\mathrm{SiO}$ ) using a turbulent width of $1.1 \mathrm{~km} \mathrm{~s}^{-1}$ for $\mathrm{L}^{2}$ Pup and $1.5 \mathrm{~km} \mathrm{~s}^{-1}$ for R Dor. Adopting a larger turbulent width means that the expansion velocity needs to be lowered somewhat in order to still provide good fits
Table 1. Source parameters obtained from modelling CO millimetre line emission.

\begin{tabular}{cccccccc}
\hline \hline Source & $\begin{array}{c}D^{a} \\
{[\mathrm{pc}]}\end{array}$ & $\begin{array}{c}L_{\star}{ }^{a} \\
{\left[L_{\odot}\right]}\end{array}$ & $\begin{array}{c}T_{\star}{ }^{a} \\
{[\mathrm{~K}]}\end{array}$ & $\begin{array}{c}\dot{M} \\
{\left[M_{\odot} \mathrm{yr}^{-1}\right]}\end{array}$ & $h$ & $\begin{array}{c}v_{\infty} \\
{\left[\mathrm{km} \mathrm{s}^{-1}\right]}\end{array}$ \\
\hline R Dor & 45 & 4000 & 2090 & $1.2 \times 10^{-7}$ & 0.05 & 5.3 & 1.5 \\
$\mathrm{~L}^{2}$ Pup & 85 & 4000 & 2690 & $2.7 \times 10^{-8}$ & 0.05 & 2.1 & 1.1 \\
\hline
\end{tabular}

${ }^{a}$ From Olofsson et al. (2002).

to the line widths. For for $\mathrm{L}^{2}$ Pup an expansion velocity of $2.1 \mathrm{~km} \mathrm{~s}^{-1}$ was found and for R Dor $5.3 \mathrm{~km} \mathrm{~s}^{-1}$. These values are about $20 \%$ lower than those presented in González Delgado et al. (2003) where a much lower microturbulent velocity of $0.5 \mathrm{~km} \mathrm{~s}^{-1}$ was adopted. Changing the prescription of the velocity field, and in particular the turbulent velocity, changes the excitation conditions and somewhat different mass loss rates are obtained. For $\mathrm{L}^{2}$ Pup a mass loss rate of $2.7 \times 10^{-8} M_{\odot} \mathrm{yr}^{-1}$ is found to provide a very good fit using an $h$-parameter of 0.05 typical of low mass loss rate objects. The $h$-parameter contain much of the properties of the dust, such as size and density of individual grains and the dust-to-gas ratio, and enters in the heating term in the energy balance equation which is solved self-consistently in the excitation analysis (see Schöier \& Olofsson 2001 for a more elaborate explanation of the $h$-parameter). For R Dor a mass loss rate of $1.2 \times 10^{-7} M_{\odot} \mathrm{yr}^{-1}$ is obtained with an $h$-parameter of 0.05 .

The resulting envelope parameters are summarized in Table 1. The distances adopted are from Olofsson et al. (2002) and are based on the assumption that the stellar luminosity is $4000 L_{\odot}$. For a discussion on this in relation to the measured Hipparcos distances, see Olofsson et al. (2002).

\subsection{SiO model}

The excitation analysis includes radiative excitation through the first vibrationally excited state. Relevant molecular data are summarized in González Delgado et al. (2003).

The abundance distribution of the circumstellar $\mathrm{SiO}$ is of importance in the radiative transfer modelling, and it is determined by two processes, dust condensation and photodissociation, as the gas expands away from the star. As outlined in more detail in González Delgado et al. (2003), the abundance is expected to decline, from the stellar atmosphere equilibrium chemistry value, once condensation of $\mathrm{SiO}$ onto dust becomes efficient when the kinetic temperature drops below the condensation temperature. This requires a minimum density which is not obtained in the low mass loss rate objects. For the higher mass loss rates the abundance declines drastically until the condensation ceases to be effective. Eventually, photodissociation sets in and this limits the size of the $\mathrm{SiO}$ envelope. Thus, to a first approximation the $\mathrm{SiO}$ abundance distribution consists of two components, a compact high abundance region close to the star, and a more extended low abundance region. The former dominates for low mass loss rates and the latter for high mass loss rates. González Delgado et al. (2003) used only one 


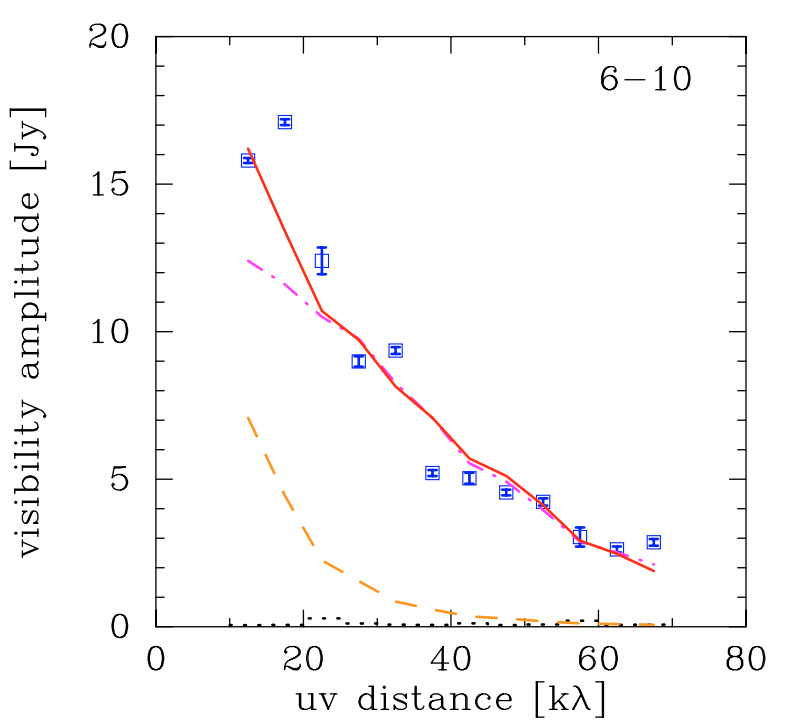

Fig. 9. Azimuthally averaged visibility amplitudes, in $5 \mathrm{k} \lambda$ bins, across the peak of the line profile $\left(6-10 \mathrm{~km} \mathrm{~s}^{-1}\right.$ in the LSR frame) for R Dor. The error bars indicate the $2 \sigma$ statistical error and does not contain the calibration uncertainty of about $15 \%$. The solid line indicates the best fit model $\left(X_{0}=4 \times 10^{-5} ; r_{\mathrm{c}}=1.2 \times 10^{15} \mathrm{~cm} ; X_{\mathrm{D}}=3 \times 10^{-6} ; r_{\mathrm{e}}=3.3 \times\right.$ $\left.10^{15} \mathrm{~cm}\right)$, the dashed line the best fit model from González Delgado et al. (2003) $\left(X_{0}=5 \times 10^{-6} ; r_{\mathrm{e}}=3.3 \times 10^{15} \mathrm{~cm}\right)$, and the dasheddotted line the best fit model without freeze-out $\left(X_{0}=5.5 \times 10^{-5} ; r_{\mathrm{e}}=\right.$ $\left.1.0 \times 10^{15} \mathrm{~cm}\right)$. The dotted histogram indicates the zero-expectation level, i.e., when no source emission is present.

component and its size was estimated from a formula obtained by fitting multi-line $\mathrm{SiO}$ data for eleven stars.

In order to investigate which $\mathrm{SiO}$ abundance distribution that best reproduces the flux picked up by the interferometer, the same $(u, v)$ sampling was applied to the predicted brightness distribution from the model envelope. Two types of abundance profiles were used (by abundance we here mean the fractional abundance of $\mathrm{SiO}$ with respect to $\mathrm{H}_{2}$ ): i) a simple Gaussian abundance profile with an inner abundance $X_{0}$ and an $e$-folding distance $r_{\mathrm{e}}$ and ii) a Gaussian profile with a depleted abundance $X_{\mathrm{D}}$ and an $e$-folding distance $r_{\mathrm{e}}$, and an added high abundance inner component $X_{0}$ introduced as a step function inside a radius $r_{\mathrm{c}}$. This provides a first approximation to the scenario outlined above where a combination of adsorption of $\mathrm{SiO}$ onto dust grains and photodissociation dictate the abundance profile.

\section{3. $R$ Dor}

The $\mathrm{SiO}$ abundance distribution used by González Delgado et al. $(2003)\left(X_{0}=5 \times 10^{-6} ; r_{\mathrm{e}}=3.3 \times 10^{15} \mathrm{~cm}\right)$ fails to explain the observed visibilities on all baselines, see the dashed line in Fig. 9. This indicates that the abundance is higher in the inner part of the envelope than what is given by the model. It should however be pointed out that the analysis of González Delgado et al. (2003) is based on a size formula for the $\mathrm{SiO}$ envelope which is obtained through multi-line fitting of data for eleven stars. A better fit is obtained for $X_{0}=5.5 \times 10^{-5}$ and $r_{\mathrm{e}}=1.0 \times 10^{15} \mathrm{~cm}$ (dash-dotted line), but all the flux is not recovered on the shortest baselines using this model. Also, the fit to the single-dish data is worse. Instead, a combination of a high abundance compact component and a low abundance more extended component $\left(X_{0}=4 \times 10^{-5} ; r_{\mathrm{c}}=1.2 \times 10^{15} \mathrm{~cm}\right.$; $\left.X_{\mathrm{D}}=3 \times 10^{-6} ; r_{\mathrm{e}}=3.3 \times 10^{15} \mathrm{~cm}\right)$ provides a very good fit to the interferometer data (solid line), and at the same time to the single-dish data as seen in Fig. 10.

Of considerable interest is to investigate the assumption made in our models of a constant velocity throughout the $\mathrm{SiO}$ line emitting region. The observed line profiles are best fitted using a somewhat lower expansion velocity of $4.9 \mathrm{~km} \mathrm{~s}^{-1}$ than obtained from the $\mathrm{CO}$ analysis $\left(5.3 \mathrm{~km} \mathrm{~s}^{-1}\right)$. This suggests that the wind has already been accelerated to within $5-10 \%$ of its terminal value at radial distances of $\approx 1 \times 10^{15} \mathrm{~cm}(\approx 30$ stellar radii). In Fig. 11 the model predictions are compared with the observations in eight velocity intervals. The model can account for the trend that the gradient in flux with baseline decreases towards the line wings. To test the sensitivity of the $\mathrm{SiO}$ line emission to the adopted velocity law a velocity gradient appropriate for a dust driven wind (e.g., Habing et al. 1994)

$v(r)=\sqrt{v_{\mathrm{i}}^{2}+\left(v_{\infty}^{2}-v_{\mathrm{i}}^{2}\right)\left(1-\frac{r_{\mathrm{i}}}{r}\right)}$

was first assumed. The ratio of the terminal wind velocity $v_{\infty}=$ $5.3 \mathrm{~km} \mathrm{~s}^{-1}$ to the velocity $v_{\mathrm{i}}$ at the inner radius $r_{\mathrm{i}}=1 \times 10^{14} \mathrm{~cm}$ was fixed to 0.25 . This velocity law provides a good fit to the line width of all available $\mathrm{SiO}$ and $\mathrm{CO}$ single-dish spectra. It was found that this velocity law only changes the results very marginally and provides an equally good fit to the observed visibilities as does a constant velocity field. Models for pulsation driven winds (Bowen 1988; Winters et al. 2000b, 2002, 2003; Struck et al. 2004) generally suggest a more slowly increasing velocity with radius. A velocity law having a constant velocity of $5.3 \mathrm{~km} \mathrm{~s}^{-1}$ outside 30 stellar radii $\left(\approx 1.4 \times 10^{15} \mathrm{~cm}\right)$ and

$v(r)=5.3\left(\frac{r}{1.4 \times 10^{15} \mathrm{~cm}}\right)^{0.5} \mathrm{~km} \mathrm{~s}^{-1}$

inside this region approximates such a slow increase. It provides equally good fits to the observations. The reason that no significant difference is observed is related to the fact that the $\mathrm{SiO} v=0, J=2 \rightarrow 1$ emission is optically thick. Thus, the emission emanates from a small region close to $r_{\mathrm{c}}$ over which no large velocity gradient exist.

A point worth mentioning is that the relatively high turbulent velocity of $1.5 \mathrm{~km} \mathrm{~s}^{-1}$ obtained for $\mathrm{R}$ Dor, as required to fit the line shapes for both the $\mathrm{CO}$ and $\mathrm{SiO}$ line emission, also significantly affects the excitation of, in particular, the lower rotational levels. In the modelling it was found that the nominal value of $0.5 \mathrm{~km} \mathrm{~s}^{-1}$ used by González Delgado et al. (2003) results in population inversions in the lower level transitions of $\mathrm{SiO}$, i.e., maser action. The line profiles in that case have strong peaks at the extreme velocities which is not observed. The higher turbulent velocity helps to quench the maser emission and produces line profiles that very closely resembles the observed ones, Fig. 10. 

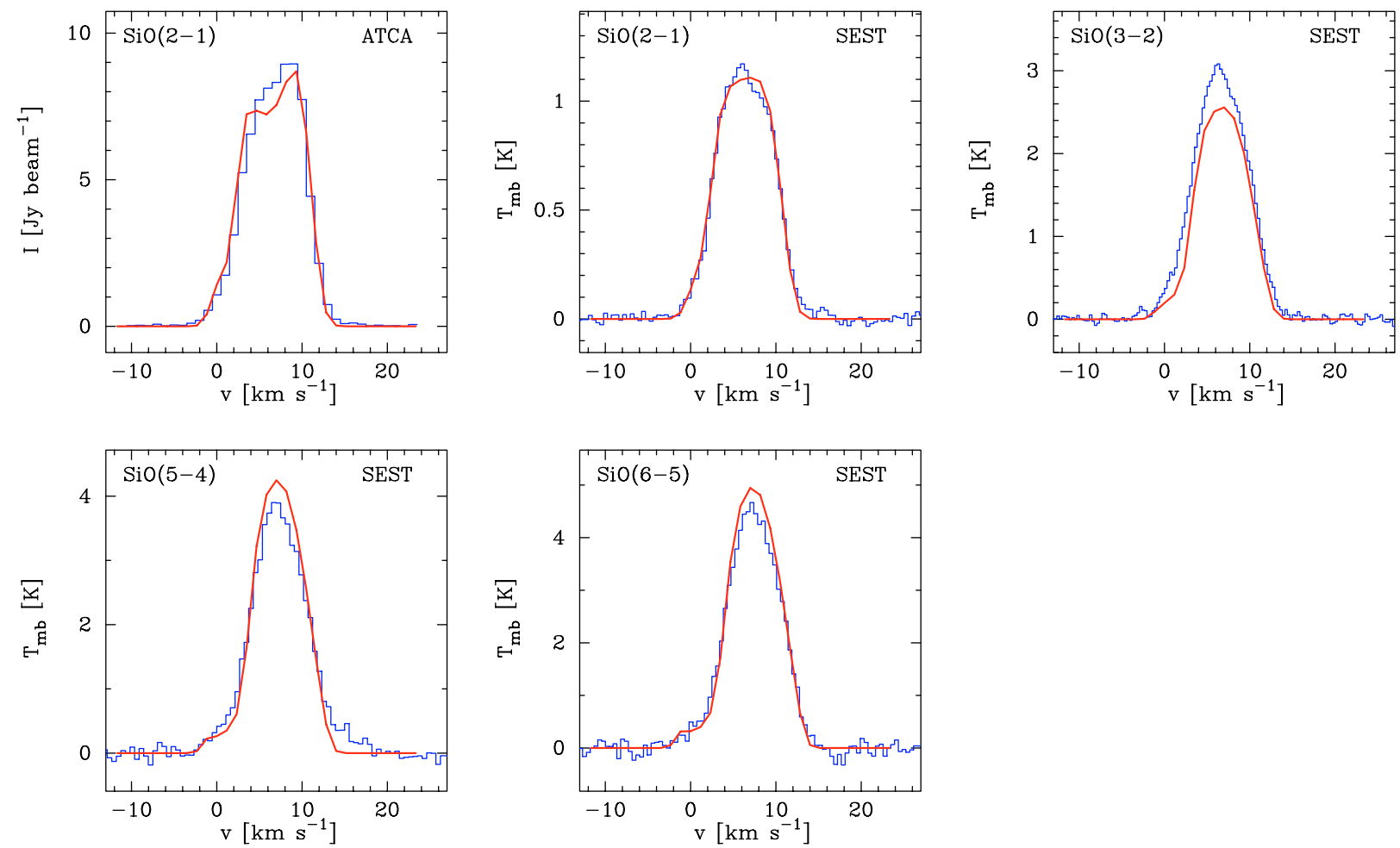

Fig. 10. Best fit model (solid line) overlayed on observations (histograms) in the form of single-dish SEST data (González Delgado et al. 2003) as well as the spectrum at the centre pixel in the cleaned ATCA map for R Dor. The velocity scale is given in the LSR frame. The calibration uncertainty in the single-dish data is about $\pm 20 \%$.

\section{4. $L^{2}$ Pup}

$\mathrm{L}^{2}$ Pup has a significantly lower (a factor of $\approx 4$ ) mass loss rate than R Dor. However, the expansion velocity of the wind is also a factor of two lower resulting in only a factor of about two lower density in the wind. Thus, the $\mathrm{SiO}$ envelope characteristics should be rather similar for the two sources.

The $\mathrm{SiO}$ abundance distribution used by González Delgado et al. $(2003)\left(X_{0}=1.4 \times 10^{-5} ; r_{\mathrm{e}}=2.1 \times 10^{15} \mathrm{~cm}\right)$ does not provide a good fit to the observed visibilities as illustrated by the dashed line in Fig. 12. While the model can account for the total flux, as indicated by the shortest baselines, it fails to account for all the flux at the longer baselines. This again indicates that the abundance is higher in the inner part of the envelope than what is given by the model. The flux seen by the interferometer at the longer baselines can be accounted for by raising $X_{0}$ to $4.5 \times 10^{-5}$, at the same time that the size of the region needs to be lowered to $1.1 \times 10^{15} \mathrm{~cm}$ in order to reproduce the total flux seen at the shorter baselines. This model is shown in Fig. 12 as the dashed-dotted line and account very well for the flux at all baselines. It should be noted that this model provides a somewhat worse fit to the single-dish data, with a reduced $\chi_{\text {red }}^{2}$ of 4.5 . In particular the high- $J$ lines come out too strong in the model by about $50 \%$ on average. In the light of the results obtained for R Dor, the combination of a high abundance compact component and a lower abundance more extended component $\left(X_{0}=6 \times 10^{-5} ; r_{\mathrm{c}}=8 \times 10^{14} \mathrm{~cm}\right.$; $\left.X_{\mathrm{D}}=2 \times 10^{-6} ; r_{\mathrm{e}}=2.1 \times 10^{15} \mathrm{~cm}\right)$ produces an equally good fit to the interferometer data (Fig. 12; solid line) and at the same time provides a much better fit to the available single-dish data $\left(\chi_{\text {red }}^{2}=1.5\right)$, Fig. 13. We note that our model can not reproduce the observed $J=5 \rightarrow 4$ line at the SEST which comes out with an integrated intensity of about $30 \%$ higher than observed. The absolute calibration at the SEST is expected to be better than approximately $\pm 20 \%$.

In Fig. 14 the model predictions are compared with the observations in four velocity intervals. The model can account well for the trend that the gradient in flux with baseline decreases towards the line wings. Note that the absolute scale of the flux in the model is somewhat lower in the wings. This is due to the fact that the model does not reproduce the line wings perfectly. Although, the source size estimated from the line wings is very well represented by the model. As for R Dor there is no evidence of any large gradients in the velocity field. Note that also for $\mathrm{L}^{2}$ Pup the $\mathrm{SiO} v=0, J=2 \rightarrow 1$ is optically thick and hence can not be expected to contain detailed information of the velocity law on smaller scales.

\section{Discussion}

\subsection{SiO abundance distribution}

Our modelling of the interferometer $\mathrm{SiO}$ data clearly show the need of an abundance distribution with a high abundance $\left(X_{0} \approx 4 \times 10^{-5}\right)$, compact $\left(\approx 1 \times 10^{15} \mathrm{~cm}\right)$ component in order to reproduce the visibilities obtained with ATCA for both $\mathrm{L}^{2}$ Pup and $\mathrm{R}$ Dor. In addition, there is evidence of a low abundance $\left(X_{\mathrm{D}} \approx 2-3 \times 10^{-6}\right)$, more extended $\left(\approx 2-3 \times 10^{15} \mathrm{~cm}\right)$ component. 


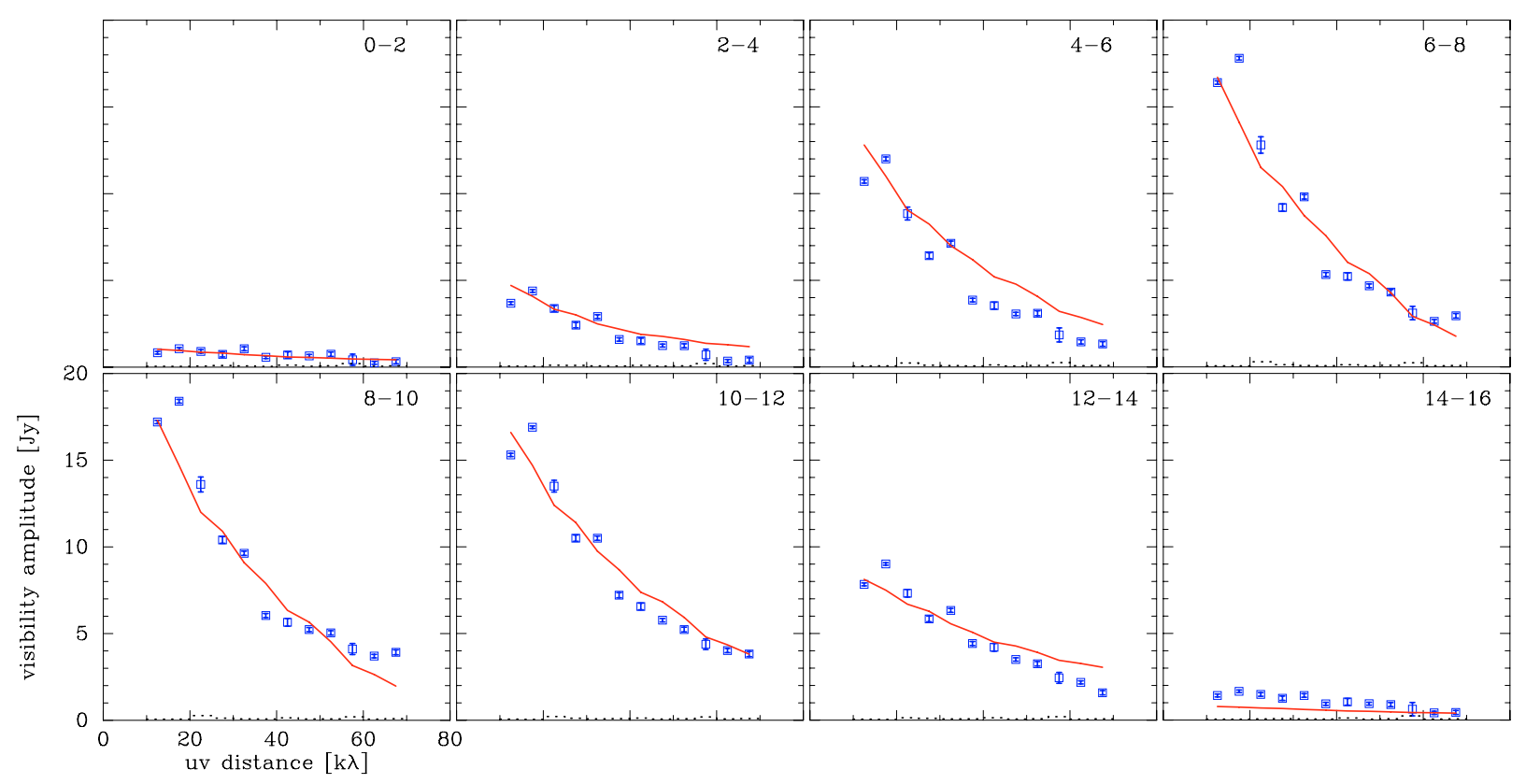

Fig. 11. Azimuthally averaged visibility amplitudes, in $5 \mathrm{k} \lambda$ bins, across various parts of the line profile (as indicated in the upper right hand corner in $\mathrm{km} \mathrm{s}^{-1}$ given in the LSR frame) for R Dor. The error bars indicate the $2 \sigma$ statistical error and does not contain the calibration uncertainty of about $15 \%$. The solid line represents the best fit model using a constant expansion velocity. The dotted histogram indicates the zero-expectation level, i.e., when no source emission is present.

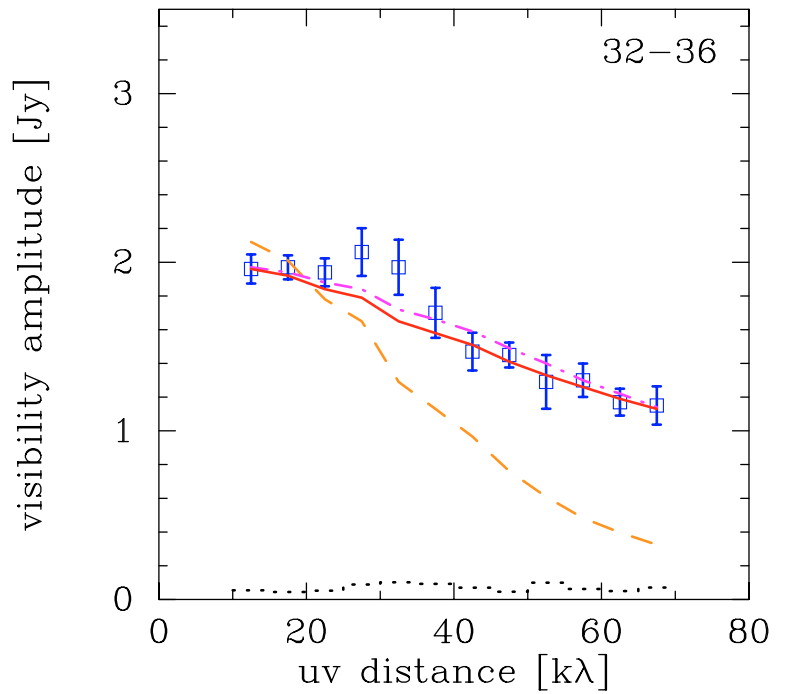

Fig. 12. Averaged visibility amplitudes, in $5 \mathrm{k} \lambda$ bins, across the peak of the line profile (32-36 $\mathrm{km} \mathrm{s}^{-1}$ in the LSR frame) for $\mathrm{L}^{2}$ Pup. The error bars indicate the $2 \sigma$ statistical error and does not contain the calibration uncertainty of about $15 \%$. The solid line indicates the best fit model $\left(X_{0}=6 \times 10^{-5} ; r_{\mathrm{c}}=8 \times 10^{14} \mathrm{~cm} ; X_{\mathrm{D}}=2 \times 10^{-6} ; r_{\mathrm{e}}=2.1 \times\right.$ $\left.10^{15} \mathrm{~cm}\right)$, the dashed line the best fit model from González Delgado et al. (2003) $\left(X_{0}=1.4 \times 10^{-5} ; r_{\mathrm{e}}=2.1 \times 10^{15} \mathrm{~cm}\right)$, and the dasheddotted line the best fit model without freeze-out $\left(X_{0}=5 \times 10^{-5} ; r_{\mathrm{e}}=\right.$ $\left.1.1 \times 10^{15} \mathrm{~cm}\right)$. The dotted histogram indicates the zero-expectation level, i.e., when no source emission is present.

This scenario is very similar to the interpretation of interferometric SiS observations of the carbon star IRC+10216 made by Bieging \& Tafalla (1993), where the abundance of SiS is seen to drastically drop by two orders of magnitudes at a radial distance of about $2 \times 10^{15} \mathrm{~cm}$.

The values of the initial $\mathrm{SiO}$ abundance derived here is very close to that expected from stellar atmosphere chemistry in thermal equilibrium, $\approx 3-4 \times 10^{-5}$ (Willacy \& Millar 1997; Duari et al. 1999). Assuming solar abundances a maximum $\mathrm{SiO}$ fractional abundance of $7 \times 10^{-5}$ is obtained (González Delgado et al. 2003). Duari et al. (1999) further showed that non-equilibrium chemistry, where the chemistry is controlled by shocks, only has a very limited effect on the abundance of $\mathrm{SiO}$ in the inner part of the wind. Thus, it is expected from theory that the photospheric abundance of $\mathrm{SiO}$ remain relatively unaffected until the combined effect of adsorption and photodissociation sets in. Our data support such a scenario. This will be discussed further in Sect. 5.2.

As an alternative explanation to the low abundances of circumstellar $\mathrm{SiO}$ that were estimated by Lambert \& vanden Bout (1978) and Morris \& Alcock (1977), Scalo \& Slavsky (1980) suggested that photodissociation by chromospheric radiation may be important. This is clearly not the case for the two sources studied here but a statistical sample needs to be investigated in order to rule out such a scenario. Unfortunately, not much is known about the chromospheres of AGB stars.

\subsection{Grain formation}

The presence of a high initial $\mathrm{SiO}$ abundance followed by a drastic decrease indicates that adsorption onto dust grains could play an important role in removing $\mathrm{SiO}$ from the gas phase. González Delgado et al. (2003) presented a relatively simple model to estimate how effectively $\mathrm{SiO}$ molecules freezeout onto dust grains. Using this we find condensation radii 

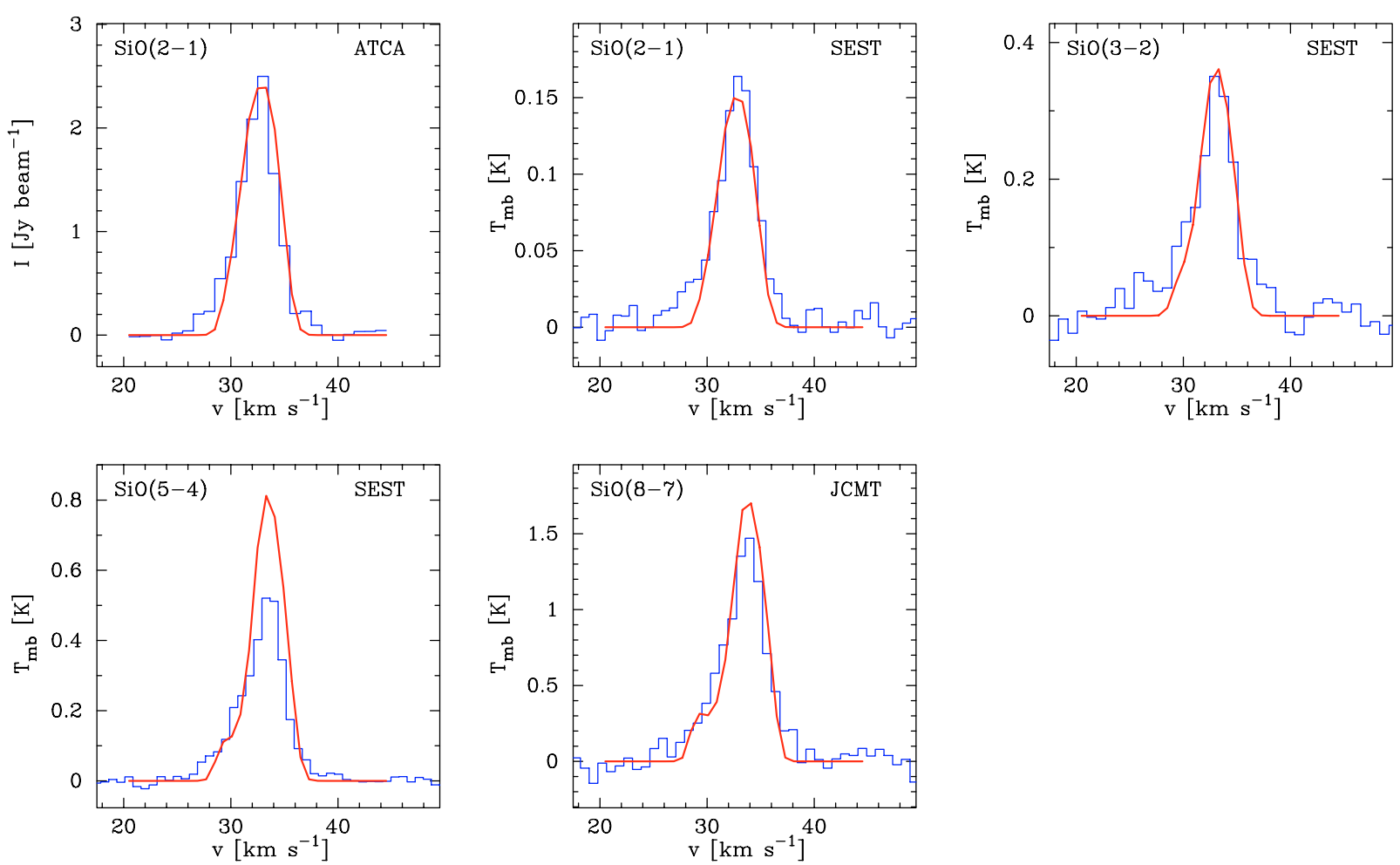

Fig. 13. Best fit model (solid line) overlayed on observations (histograms) in the form of single-dish SEST (González Delgado et al. 2003) and JCMT data (unpublished data) as well as the spectrum at the centre pixel in the cleaned ATCA map for $\mathrm{L}^{2}$ Pup. The velocity scale is given in the LSR frame. The calibration uncertainty in the single-dish data is about $\pm 20 \%$.

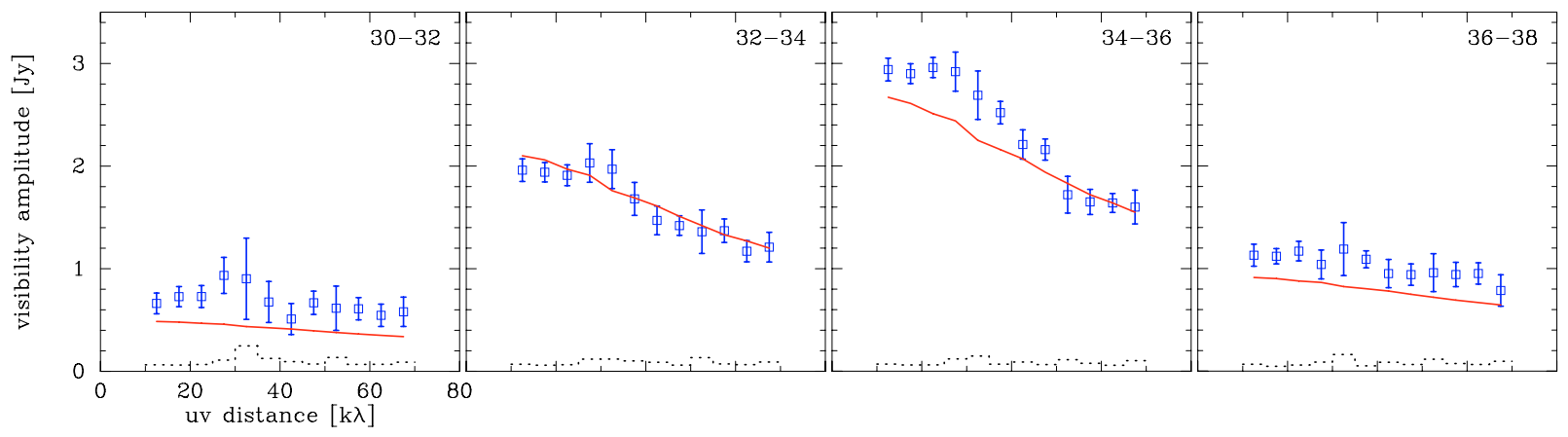

Fig. 14. Averaged visibility amplitudes, in $5 \mathrm{k} \lambda$ bins, across various parts of the line profile (as indicated in the upper right hand corner in $\mathrm{km} \mathrm{s}^{-1}$ given in the LSR frame) for $\mathrm{L}^{2}$ Pup. The error bars indicate the $2 \sigma$ statistical error and does not contain the calibration uncertainty of about $15 \%$. The solid line represents the best fit model using a constant expansion velocity. The dotted histogram indicates the zero-expectation level, i.e., when no source emission is present.

of $\approx 5 \times 10^{14} \mathrm{~cm}$ for both stars (essentially determined by where the dynamical time scale equals the evaporation time). The degree of adsorption critically depends on the amount of dust available. For the dust parameters hinted at from the CO modelling, and contained in the $h$-parameter, we expect the $\mathrm{SiO}$ abundance to drop by about a factor of $\sim 3$ beyond this radius (for both stars). Likewise, we can use the simple photodissociation model outlined in González Delgado et al. (2003) to derive $\mathrm{SiO}$ photodissociation radii of about $10^{15}$ and $3 \times 10^{15} \mathrm{~cm}$ for $\mathrm{L}^{2}$ Pup and R Dor, respectively. Thus, within the considerable uncertainties, we expect the $\mathrm{SiO}$ line emission to be strongly dominated by the compact high abundance region in $\mathrm{L}^{2}$ Pup. In the case of $\mathrm{R}$ Dor the low abundance region between the condensation and photodissociation radii will also contribute. Our observational results are certainly consistent with this, even though the estimated abundance drop beyond the condensation radius is larger than predicted by the model. Given the simplicity of the treatment of the adsorption process and the uncertainty in the adopted dust parameters, we find this acceptable. More elaborate chemical models than the ones presented by González Delgado et al. (2003) are needed to take the combined effect of adsorption and photodissociation properly into account.

In addition, as indicated in Sect. 5.1 the role of chromospheric radiation as a means of dissociating $\mathrm{SiO}$ in these stars needs to be investigated. Scalo \& Slavsky (1980) suggested that $\mathrm{SiS}$ might be the best probe of the degree of adsorption of $\mathrm{Si}$ bearing molecules since it can be readily formed though 
reactions with $\mathrm{H}$ and $\mathrm{H}_{2}$ in the inner wind. In contrast there is no easy means of forming $\mathrm{SiO}$. However, this was based on the assumption that all Si-molecules were destroyed by chromospheric radiation. Nevertheless, observations of $\mathrm{SiS}$ is an important complement to the $\mathrm{SiO}$ observations, and it has been detected in O-rich envelopes (Lindqvist et al. 1988; Olofsson et al. 1998).

\subsection{Wind dynamics}

The success of the modelling using a constant velocity (and a higher micro-turbulent velocity width) strongly suggests that the wind has reached its terminal velocity already within $\sim 20-30$ stellar radii. This is further supported by the fact that the $\mathrm{SiO}$ and $\mathrm{CO}$ line data can be modelled using the same expansion velocity (within 5-10\%). The marked difference in shapes between the $\mathrm{SiO}$ and the $\mathrm{CO}$ lines is attributed to strong self absorption of emission in the part of the wind moving towards the observer in the case of SiO. Thus, we support the conclusion by Sahai \& Bieging (1993) rather than that of Lucas et al. (1992) where an extended region of acceleration was advocated, but also note that we have so far studied only two low mass loss rate objects.

It has been suggested from hydrodynamical calculations (Bowen 1988; Winters et al. 2000b, 2002, 2003; Struck et al. 2004) that in very low mass loss rate $\left(\dot{M} \lesssim 5 \times 10^{-7} M_{\odot} \mathrm{yr}^{-1}\right)$, low expansion velocity $\left(\$ 5 \mathrm{~km} \mathrm{~s}^{-1}\right)$ objects, such as R Dor and $\mathrm{L}^{2}$ Pup, the main driving mechanism behind these tenuous winds is stellar pulsation, and the wind acceleration is low. This is in contrast to winds which reach higher mass loss rates and expansion velocities where dust plays a primary role, and where the wind acceleration is very efficient. From the present data we can not distinguish between these competing scenarios mainly due to the $\mathrm{SiO}$ emission being optically thick, and therefore most of the emission emanates from a region where the wind is close to the terminal velocity. Observations of optically thin SiS lines could possibly be useful to probe the velocity field. However, the SiS line emission is expected to be more than an order of magnitude weaker than that of $\mathrm{SiO}$.

\subsection{Predictions for high-J SiO lines}

Presented here in Table 2 are estimated line intensities for selected transitions of $\mathrm{SiO}$ based on our models for $\mathrm{R}$ Dor using a $12 \mathrm{~m}$ telescope. The differences between the one-component model derived by González Delgado et al. (2003), and the new models required to fit the interferometer data presented here, increase as higher frequency transitions are considered. This shows the potential of single-dish telescopes such as CSO, JCMT and the upcoming APEX ${ }^{3}$ and ASTE telescopes to study the properties of stellar winds close to the central star.

\footnotetext{
${ }^{3}$ The Atacama Pathfinder EXperiment (APEX), is a collaboration between Max Planck Institut für Radioastronomie (in collaboration with Astronomisches Institut Ruhr Universität Bochum), Onsala Space Observatory and the European Southern Observatory (ESO) to construct and operate a modified ALMA prototype antenna as a single dish on the high altitude site of Llano Chajnantor.
}

Table 2. Predicted SiO line intensities (integrated over the line and in main-beam brightness scale using a $12 \mathrm{~m}$ telescope) towards R Dor for selected rotational transitions.

\begin{tabular}{crrccc}
\hline \hline Transition & $\begin{array}{c}v \\
{[\mathrm{GHz}]}\end{array}$ & $\begin{array}{c}\theta_{\mathrm{mb}} \\
{\left[{ }^{\prime \prime}\right]}\end{array}$ & $\begin{array}{c}\text { Model 1 } \\
{\left[\mathrm{K} \mathrm{km} \mathrm{s}^{-1}\right]}\end{array}$ & $\begin{array}{c}\text { Model 2 } \\
{\left[\mathrm{K} \mathrm{km} \mathrm{s}^{-1}\right]}\end{array}$ & $\begin{array}{c}\text { Model 3 } \\
{\left[\mathrm{K} \mathrm{km} \mathrm{s}^{-1}\right]}\end{array}$ \\
\hline $5 \rightarrow 4$ & 217.1 & 29 & 20 & 19 & 24 \\
$8 \rightarrow 7$ & 347.3 & 18 & 28 & 33 & 39 \\
$11 \rightarrow 10$ & 477.5 & 13 & 30 & 46 & 55 \\
$16 \rightarrow 15$ & 694.1 & 9 & 28 & 58 & 63 \\
$20 \rightarrow 19$ & 867.5 & 7 & 27 & 65 & 63 \\
$30 \rightarrow 29$ & 1300.5 & 5 & 15 & 52 & 64 \\
\hline
\end{tabular}

${ }^{a}$ Best fit model from González Delgado et al. (2003) $\left(X_{0}=5 \times 10^{-6}\right.$; $\left.r_{\mathrm{e}}=3.3 \times 10^{15} \mathrm{~cm}\right)$.

${ }^{b}$ High abundance compact component $\left(X_{0}=5.5 \times 10^{-5} ; r_{\mathrm{e}}=1.0 \times\right.$ $\left.10^{15} \mathrm{~cm}\right)$.

${ }^{c}$ Best fit model $\left(X_{0}=4 \times 10^{-5} ; r_{\mathrm{c}}=1.2 \times 10^{15} \mathrm{~cm} ; X_{\mathrm{D}}=3 \times 10^{-6}\right.$; $\left.r_{\mathrm{e}}=3.3 \times 10^{15} \mathrm{~cm}\right)$.

APEX and ASTE are of particular interest since they will eventually open up the $\mathrm{THz}$ region for observations. They will also be the only telescopes at the southern hemisphere being able to observe at sub-milimetre wavelengths.

\section{Conclusions}

High resolution, interferometric, millimetre observations at $\approx 1^{\prime \prime}$ resolution of $\mathrm{SiO} v=0, J=2 \rightarrow 1$ line emission towards the two O-rich AGB stars $\mathrm{L}^{2}$ Pup and R Dor have been performed. The emission is resolved, very centrally peaked, and suggests an overall spherical symmetry, even though there is an indication of a departure from spherical symmetry in the case of R Dor at the arcsecond scale. A detailed excitation analysis was performed suggesting that the $\mathrm{SiO}$ abundance is very high $\left(\approx 4 \times 10^{-5}\right)$ in the inner part $\left(\lesssim 1 \times 10^{15} \mathrm{~cm}\right)$ of the circumstellar envelopes around both stars, consistent with predictions from LTE stellar atmosphere chemistry. For R Dor the interferometer data further suggests that there is an additional, more extended, component with a significantly lower $\mathrm{SiO}$ abundance. We interpret this as the result of effective adsorption of $\mathrm{SiO}$ onto dust grains. Such a conclusion is less clear in the case of $\mathrm{L}^{2} \mathrm{Pup}$.

A comparison of model and observed line profiles further suggests that micro-turbulent motions are of the order of $1-1.5 \mathrm{~km} \mathrm{~s}^{-1}$. This is a significant fraction of the wind velocity in these slowly expanding winds $\left(\$ 5 \mathrm{~km} \mathrm{~s}^{-1}\right)$, and it produces strong self absorption of the blue-shifted emission giving the $\mathrm{SiO}$ line profiles their characteristic shape. Additionally, the interferometer data provide constraints on the size of the region in which the wind is being accelerated. It is found that a constant velocity over the $\mathrm{SiO}$ emitting region can successfully account for the visibility amplitudes obtained at different velocity intervals. Furthermore, it is possible to model the line shapes of both $\mathrm{SiO}$ and $\mathrm{CO}$ emission using the same expansion velocity (within 5-10\%) for both our sources. This constrains the acceleration region to within $\$ 20-30$ stellar radii.

We conclude that $\mathrm{SiO}$ line emission plays a very important role in the study of circumstellar envelopes, both with respect 
to its dynamics and the gas and grain chemistry. However, it should be remembered that both sources studied here have very low mass loss rates and expansion velocities and that a more representative sample of O-rich sources needs to be studied in order to solidify the conclusion reached here. This could also be used to investigate the role of chromospheric radiation in photodissociation of $\mathrm{SiO}$ molecules. Such an effort is currently underway, and it justifies the development of a more elaborate model to describe the abundance distribution of $\mathrm{SiO}$.

Acknowledgements. F.L.S., H.O. and M.L. are grateful to The Swedish Research council for financial support. T.W. is supported by an ARC-CSIRO Linkage Grant to the University of New South Wales.

\section{References}

Bernes, C. 1979, A\&A, 73, 67

Bieging, J. H., \& Tafalla, M. 1993, AJ, 105, 576

Bowen, G. H. 1988, ApJ, 329, 299

Bujarrabal, V., Planesas, P., Martin-Pintado, J., Gomez-Gonzalez, J., \& del Romero, A. 1986, A\&A, 162, 157

Cotton, W. D., Mennesson, B., Diamond, P. J., et al. 2004, A\&A, 414, 275

Danchi, W. C., Bester, M., Degiacomi, C. G., Greenhill, L. J., \& Townes, C. H. 1994, AJ, 107, 1469

Duari, D., Cherchneff, I., \& Willacy, K. 1999, A\&A, 341, L47

González Delgado, D., Olofsson, H., Kerschbaum, F., et al. 2003, A\&A, 411, 123

Högbom, J. A. 1974, A\&AS, 15, 417

Habing, H. J., Tignon, J., \& Tielens, A. G. G. M. 1994, A\&A, 286, 523

Keady, J. J., \& Ridgway, S. T. 1993, ApJ, 406, 199
Kerschbaum, F., \& Olofsson, H. 1999, A\&AS, 138, 299

Lambert, D. L., \& vanden Bout, P. A. 1978, ApJ, 221, 854

Lindqvist, M., Nyman, L.-Å., Olofsson, H., \& Winnberg, A. 1988, A\&A, 205, L15

Lindqvist, M., Schöier, F. L., Lucas, R., \& Olofsson, H. 2000, A\&A, 361, 1036

Lucas, R., Bujarrabal, V., Guilloteau, S., et al. 1992, A\&A, 262, 491

Morris, M., \& Alcock, C. 1977, ApJ, 218, 687

Olofsson, H., González Delgado, D., Kerschbaum, F., \& Schöier, F. L. 2002, A\&A, 391, 1053

Olofsson, H., Lindqvist, M., Nyman, L.-Å., \& Winnberg, A. 1998, A\&A, 329, 1059

Sahai, R., \& Bieging, J. H. 1993, AJ, 105, 595

Sault, R. J., Teuben, P. J., \& Wright, M. C. H. 1995, in ASP Conf. Ser., 77, Astronomical Data Analysis Software and Systems IV, 4, 433

Scalo, J. M., \& Slavsky, D. B. 1980, ApJ, 239, L73

Schöier, F. L., \& Olofsson, H. 2001, A\&A, 368, 969

Struck, C., Smith, D. C., Willson, L. A., Turner, G., \& Bowen, G. H. 2004, MNRAS, submitted

van Zadelhoff, G.-J., Dullemond, C. P., van der Tak, F. F. S., et al. 2002, A\&A, 395, 373

Willacy, K., \& Millar, T. J. 1997, A\&A, 324, 237

Winters, J. M., Keady, J. J., Gauger, A., \& Sada, P. V. 2000a, A\&A, 359,651

Winters, J. M., Le Bertre, T., Jeong, K. S., Helling, C., \& Sedlmayr, E. 2000 b, A\&A, 361, 641

Winters, J. M., Le Bertre, T., Nyman, L.-Å., Omont, A., \& Jeong, K. S. 2002, A\&A, 388, 609

Winters, J. M., Le Bertre, T., Jeong, K. S., Nyman, L.-Å., \& Epchtein, N. 2003, A\&A, 409, 715

Wong, T., Schöier, F. L., Lindqvist, M., \& Olofsson, H. 2004, A\&A, 413, 241

Zubko, V., \& Elitzur, M. 2000, ApJ, 544, L137 